\title{
Covid-19 and development of heart failure: mystery and truth
}

\author{
Hope Onohuean ${ }^{1}$ (D) Hayder M. Al-kuraishy ${ }^{2}$ - Ali I. Al-Gareeb ${ }^{2}$. Safaa Qusti ${ }^{3}$ - Eida M. Alshammari ${ }^{4}$. \\ Gaber El-Saber Batiha ${ }^{5}$
}

Received: 7 July 2021 / Accepted: 26 August 2021 / Published online: 4 September 2021

(c) The Author(s), under exclusive licence to Springer-Verlag GmbH Germany, part of Springer Nature 2021

\begin{abstract}
Coronavirus disease 2019 (Covid-19) is a novel worldwide pandemic caused by a novel severe acute respiratory syndrome coronavirus 2 (SARS-CoV-2). During Covid-19 pandemic, socioeconomic deprivation, social isolation, and reduced physical activities may induce heart failure (HF), destabilization, and cause more complications. HF appears as a potential hazard due to SARS-CoV-2 infection, chiefly in elderly patients with underlying comorbidities. In reality, the expression of cardiac ACE2 is implicated as a target point for SARS-CoV-2-induced acute cardiac injury. In SARS-CoV-2 infection, like other febrile illnesses, high blood viscosity, exaggerated pro-inflammatory response, multisystem inflammatory syndrome, and endothelial dysfunction-induced coagulation disorders may increase risk of HF development. Hypoxic respiratory failure, as in pulmonary edema, severe acute lung injury (ALI), and acute respiratory distress syndrome (ARDS) may affect heart hemodynamic stability due to the development of pulmonary hypertension. Indeed, Covid-19-induced HF could be through the development of cytokine storm, characterized by high proliferation pro-inflammatory cytokines. In cytokine storm-mediated cardiac dysfunction, there is a positive correlation between levels of pro-inflammatory cytokine and myocarditis-induced acute cardiac injury biomarkers. Therefore, Covid-19-induced HF is more complex and related from a molecular background in releasing pro-inflammatory cytokines to the neuro-metabolic derangements that together affect cardiomyocyte functions and development of HF. Anti-heart failure medications, mainly digoxin and carvedilol, have potent anti-SARS-CoV-2 and anti-inflammatory properties that may mitigate Covid-19 severity and development of HF. In conclusion, SARS-CoV-2 infection may lead to the development of HF due to direct acute cardiac injury or through the development of cytokine storms, which depress cardiomyocyte function and cardiac contractility. Anti-heart failure drugs, mainly digoxin and carvedilol, may attenuate severity of HF by reducing the infectivity of SARS-CoV-2 and prevent the development of cytokine storms in severely affected Covid-19 patients.
\end{abstract}

Keywords Covid-19 $\cdot$ SARS-CoV-2 $\cdot$ Development $\cdot$ Heart failure

Hope Onohuean

hope.onohuean1@kiu.ac.ug; onohuean@gmail.com

Gaber El-Saber Batiha

gaberbatiha@gmail.com;

dr_gaber_batiha@vetmed.dmu.edu.eg

Hayder M. Al-kuraishy

Haydermutter@uomustansiriyah.edu.iq

Ali I. Al-Gareeb

dr.alialgareeb@uomustansiriyah.edu.iq

Safaa Qusti

squsti@kau.edu.sa

Eida M. Alshammari

eida.alshammari@uoh.edu.sa
1 Department of Pharmacology and Toxicology, Biopharmaceutics Unit, School of Pharmacy, Kampala International University, Western-Campus, Kampala, Uganda

2 Department of Clinical Pharmacology and Medicine, College of Medicine, ALmustansiriyia University, Baghdad, Iraq

3 Biochemistry Department, Faculty of Science, King Abdulaziz University, Jeddah, Saudi Arabia

4 Department of Chemistry, College of Sciences, University of Ha'il, Ha'il, Saudi Arabia

5 Department of Pharmacology and Therapeutics, Faculty of Veterinary Medicine, Damanhour University, Damanhour, AlBeheira 22511, Egypt 


\section{Background}

Coronavirus disease 2019 (Covid-19) is a novel worldwide pandemic caused by a novel severe acute respiratory syndrome coronavirus 2 (SARS-CoV-2), which was primarily recognized in Wuhan, China, according to the initial epidemiological finding (Lugnier et al. 2021). Covid-19 affects millions of people, and up to late May 2021, the number of infected people reaches more than 160 million. Though greatest of affected people are asymptomatic or presented with mild symptoms in $80 \%$, however, $15-20 \%$ of affected patients require hospitalization due to development of acute lung injury (ALI) and/or respiratory distress syndrome (ARDS) (Al-kuraishy et al. 2021b). Severe Covid-19 cases are linked to hyper-inflammations and the development of cytokine storms (Al-kuraishy et al. 2021a).

The leading causes of inflammatory and immunological disorders in patients with critical Covid-19 are related to the overactivation of $\mathrm{T}$ cells and macrophages following the release of massive pro-inflammatory cytokines such as interleukins and chemokines (Al-kuraishy et al. 2021e). Mainly, IL-6, IL1 $\beta$, IL-8, and tumor necrosis factor-alpha $(\mathrm{TNF}-\alpha)$ via severe SARS-CoV-2 infections are linked with the development of ALI/ARDS and multi-organ failure (MOF) (Al-Kuraishy et al. 2020). One of the most significant probable entry-point of SARS-CoV-2 is the angiotensin-converting enzyme 2 (ACE2). The interaction between SARS-CoV-2 and ACE2 leads to noteworthy lessening of this anti-inflammatory receptor. ACE2 is involved in the control of the renin-angiotensin system (RAS) via conversion of vasoconstrictor angiotensin II (Ang II) into vasodilator anti-inflammatory Ang 1-7 and Ang 1-9 (Al-Kuraishy et al. 2020). Therefore, downregulation of ACE2 and advancement of circulating AngII during SARS-CoV-2 infection could be the possible mechanism overdue initiation of inflammatory instabilities (Moubarak et al. 2021).

Heart failure (HF) is defined as failure of heart to maintain sufficient blood flow for body tissue. Patients with HF experience exertional dyspnea, leg edema, and excessive tiredness. HF is divided to right side HF, left side HF, and congestive HF. The leading causes of HF are hypertension, ischemic heart disease, arrhythmia, cardiomyopathy, and myocarditis (Rossignol et al. 2019). HF represents one of the common cardiovascular disorders in occidental counties; it affects $2 \%$ of the adult population that increases to $10 \%$ over the age of 65 years; this rate may increase due to expanding life span and improvement of survival rates from associated risk factors of HF (Farré et al. 2017).

During the Covid-19 pandemic, socioeconomic deprivation, social isolation, and reduced physical activities may induce HF destabilization and cause more complications.
Besides, SARS-CoV-2 infection and related inflammatory burden could aggravate preexistent HF or induces newonset HF (Reza et al. 2020). In this sense, this mini-review aimed to highlighted and shed light on the pragmatic association between SARS-CoV-2 infection and development of HF in Covid-19 patients regarding metabolic and inflammatory points.

\section{Viral infections and risk of heart failure}

It has been reported that different viral infections may induce development of HF; Butt et al. (2011) illustrated that human immune deficiency virus (HIV) infection is regarded as a risk factor for induction of HF due to immunological reaction-induced myocarditis, secondary infections, nutritional deficiency, and negative impact of antiretroviral drugs on the heart (Butt et al. 2011). Of note, about $50 \%$ of patients with HIV infection have diastolic dysfunction and myocarditis in 50\% of HIV infection patients (Nico et al. 2010). In contrast, Kristoffersen et al., radionuclide ventriculography study of HIV infection patients, did not find any evidence of ventricular dysfunction (Kristoffersen et al. 2008).

Hepatitis $\mathrm{C}$ virus (HCV) could prompt progression of $\mathrm{HF}$ by causing myocarditis and acute cardiac injury, as evident by a positive correlation between biomarkers of cardiac injury and anti-HCV antibodies (Matsumori et al. 2006). Similarly, there is a strong association between anti-HCV antibodies and HF-associated inflammatory biomarkers in seropositive patients for HCV (Tsui et al. 2009). Adinolfi et al. explore that chronic $\mathrm{HCV}$ infection can cause $\mathrm{HF}$ through the progression of coronary atherosclerotic plaque (Adinolfi et al. 2014). Moreover, chronic HCV infection and used antiviral agents may increase the risk of hospitalization of patients with complicated HF due to the development of dilated cardiomyopathy (Lin et al. 2018).

Furthermore, infection with a respiratory syncytial virus (RSV) often leads to development of HF due to underlying cardiopulmonary and immunoprophylaxis disorders (Falsey et al. 2019). RSV also led to various cardiovascular disorders such as ischemic changes due to coronary plaque destabilization and HF through induction of different pro-inflammatory cytokines, adversely affecting heart function (Falsey et al. 2019).

During the influenza epidemic, the hospitalization rate for patients with HF is dramatically increased, suggesting a temporal association between viral infection and cardiomyocyte injury (Vardeny and Solomon 2019). The potential interplay between influenza infection and the development of HF is related to different pathophysiological disturbances, including hypoxemia, neuroendocrine and sympathetic activation, cardio-renal injury-induced volume overload, direct cardiomyocyte injury, and associated hyperinflammatory 
that interacted mutually in the progress of HF (Panhwar et al. 2019).

In consideration of these findings, different viral infections in early and late stages may cause progression of HF through the direct viral effect or indirectly through activated pro-inflammatory cytokines. Viral infections induce hypoxemia, sympathetic activation, release of pro-inflammatory cytokines, and cardiomyocyte injury (Chen and Hao 2020). Sympathetic activation activates the renin-angiotensin system (RAS) with induction of cardio-renal syndrome and volume overload. In addition, pro-inflammatory cytokines and RAS are interacted together in the development of the cardio-renal syndrome and reduction of cardiac contractility. These pathophysiological changes promote the development of HF (Soto et al. 2017) (Fig. 1).

\section{Covid-19 and risk of heart failure}

With the progress of Covid-19 menacing, HF appears as a potential hazard due to SARS-CoV-2 infection chiefly in the elderly patients with underlying comorbidities such as hypertension, ischemic heart disease, and diabetes mellitus (Bader et al. 2021). It has been shown that cardiac injury biomarkers are increased in patients with severe Covid-19 suggesting that development of direct acute cardiac injury and/or myocarditis or indirect due to pulmonary complications such as ALI and ARDS (Zhou et al. 2020). It has been hypothesized that early SARS-CoV-2 infection leads to a classic form of $\mathrm{HF}$ with preserved ejection fraction due to acute cardiac injury, whereas in the later phase of SARS$\mathrm{CoV}-2$ infection, HF with systolic dysfunction is developed due to progression of cytokine storm (Peng et al. 2021).

In reality, the expression of cardiac ACE2 is implicated as a target point for SARS-CoV-2-induced acute cardiac injury. Expression of cardiac ACE2 is at pericytes, mainly in patients with HF that increase the risk of a heart attack during SARS-CoV-2 infection (Chen et al. 2020b). Different interwoven and contested articles implicate angiotensin-converting enzyme inhibitors (ACEIs) and angiotensin receptor blockers (ARBs) in the enhancement of SARS-CoV-2 infection through overexpression of cardiac ACE2 (Patel and Verma 2020; Sanchis-Gomar et al. 2020). Previously, different studies highlighted the protective role of ACE2 against the development of HF, atherosclerosis, and endothelial dysfunction (Lovren et al. 2008; Wang et al. 2013). Ohtsuki et al. (2010) confirmed that expression of ACE2 improves cardiomyocytes remodeling in patients with end-stage left ventricular HF (Ohtsuki et al. 2010). Therefore, cardiac ACE2 is regarded as an adaptive mechanism retarding the progression of left ventricular remodeling. Likewise, Keidar et al. (2005) illustrated that aldosterone antagonist eplerenone improves cardiac contractility through augmentation expression of ACE2, which increase the protective Ang1-7 and reduces harmful AngII (Keidar et al. 2005). These findings may explain the protective role of ACEIs and ARBs against SARS-CoV-2 infection-induced acute cardiac injury through overexpression of cardiac ACE2 (Alkuraishy et al. 2021d). Moreover, in HF, chymase activity is increased, leading to elevation of AngII and reduction of

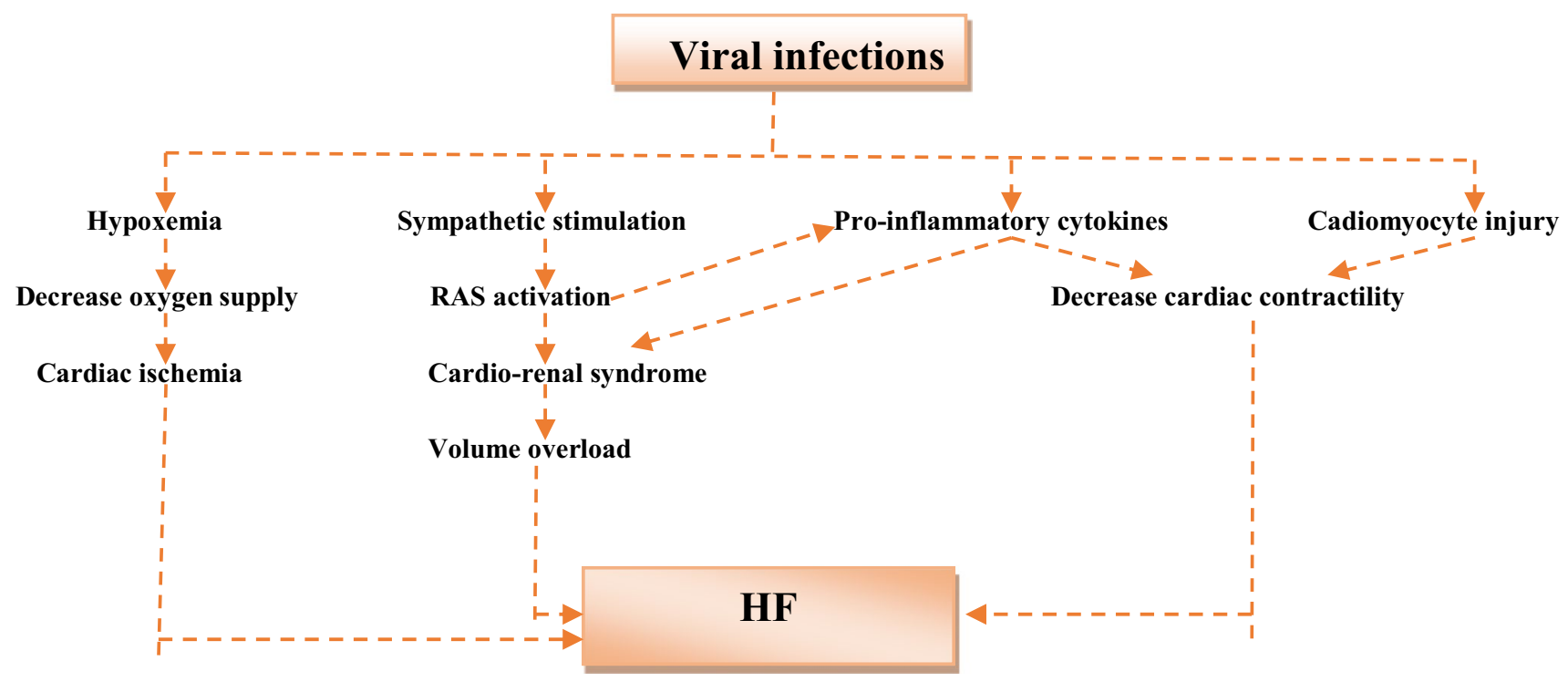

Fig. 1 Role viral infections in the development of heart failure (HF): viral infections induce hypoxemia, sympathetic activation, release of pro-inflammatory cytokines, and cardiomyocyte injury. Sympathetic activation activates renin-angiotensin system (RAS) with induction of cardio-renal syndrome and volume overload. In addition, pro-inflammatory cytokines and RAS interacted together in the development of cardio-renal syndrome and reduction of cardiac contractility. These pathophysiological changes promote development of HF 
Ang 1-7. As well, administration of recombinant ACE2 will normalize AngII/Ang 1-7 ratio and could be a potential therapeutic modality for HF (Al-kuraishy et al. 2021c). Herein, cessation of ACEIs and ARBs in an early phase of Covid-19 may destabilize cardio-metabolic response and aggravate $\mathrm{HF}$ and cardiovascular complications (Bozkurt et al. 2020). In the SARS epidemic, viral RNA of SARS-CoV was observed in 35\% of autopsy from human heart SARS epidemic in Toronto; these autopsies characterized by myocyte necrosis, macrophage infiltration, and reduction of ACE2 expression (Oudit et al. 2009).

In SARS-CoV-2 infection, like other febrile illnesses, high blood viscosity, exaggerated pro-inflammatory response, multisystem inflammatory syndrome, and endothelial dysfunction-induced coagulation disorders may increase risk of HF (Belhadjer et al. 2020). Hypoxic respiratory failure, as in pulmonary edema, severe ALI, and ARDS may affect heart hemodynamic stability due to the development of pulmonary hypertension (Taz et al. 2021). Indeed, Covid-19-induced HF might be through the development of cytokine storm, which is characterized by high proinflammatory cytokines such as IL-1 $\beta$, IL-6, and monocyte chemoattractant protein-1 (MCP-1) that lead to fulminant myocarditis (Chen et al. 2020a). There is a positive correlation between pro-inflammatory cytokine levels and biomarkers of myocarditis-induced acute cardiac injury (Saed Aldien et al. 2021). As well, SARS-CoV-2 infection-induced hyperferritinemia may lead to acute cardiac dysfunction, HF, and cardiac arrest through augmentation of inflammatorymediated cardiomyocyte injury (VasanthiDharmalingam et al. 2021). Ashraf et al., a case-report study, illustrated that high ferritin serum level in women with Hodgkin lymphoma is associated with development of HF (Ashraf et al. 2011).

In line with the large body of literature that confirmed the association between cytokine storm and risk of HF, IL-6 is regarded as a prototype of pro-inflammatory cytokine linked with the progression of ventricular dysfunction and congestive HF (Al-Kuraishy and Al-Gareeb 2017). A retrospective cohort study involving 2329 patients with HF showed that IL-6 serum is increased in 50\% of patients and predicts mortality (Markousis-Mavrogenis et al. 2019). Therefore, IL-6 could be a potential link between SARS-CoV-2 infection and development of HF since IL-6 serum level is associated with Covid-19 severity and impairment of T cell cytotoxic activity (Han et al. 2020). In addition, high TNF- $\alpha$ level in severe Covid-19 may be associated with HF development since TNF- $\alpha$ level is correlated with biomarkers of cardiomyocyte injury (Al-kuraishy et al. 2018; Noroozi et al. 2020).

During early SARS-CoV-2 infection, both nods like receptor pyrin 3 (NLRP3) inflammasome and NF- $\mathrm{KB}$ signaling pathway are activated directly by SARS-CoV-2 viral proteins leading to the bursting of pro-inflammatory cytokines (Hemmat et al. 2021). It has been reported that NLRP3 inflammasome has a vital role in the progression of $\mathrm{HF}$ through activation of systemic inflammatory milieu (Butts et al. 2015). Also, hyperactivity of the NF- $\kappa B$ signaling pathway is associated with the development of cardiac dysfunction and progression of $\mathrm{HF}$ (Al-Kuraishy and Al-Gareeb 2016). Therefore, stimulation of NLRP3 inflammasome and NF- $\mathrm{BB}$ signaling pathway by SARS-CoV-2 viral proteins could be the causal relationship between Covid-19 severity and progression of HF. In addition, p38 mitogen-activated protein kinase (MAPK) is highly activated by SARS-CoV-2 and linked with Covid-19 severity and systemic complications such as HF and cytokine storm (Meijles et al. 2020; Asiedu et al. 2021).

Taken together, these inflammatory mediators may cause sequential impeding of cardiac contractility during early and late phases of SARS-CoV-2 infection. Besides, hyperinflammation and hypercytokinemia during cytokine storm may depress cardiomyocyte functions through the development of severe myocarditis (Imazio et al. 2020).

It has been shown in experimental studies that high circulating AngII may lead to HF due to direct cardiomyocyte injury through AT1R and upregulation of distinct stretchactivated channels (Piezol 1 receptor), which involved in ventricular remodeling (Liang et al. 2017). High AngII in SARS-CoV-2 infection due to downregulation of ACE2 could be a proposed mechanism of HF in severe Covid-19. Thus, attenuation of AngII by ARBs or ACEIs may reduce Covid-19 severity and development of HF (Matsoukas et al. 2021). Indeed, RAS is also involved in metabolic derangement and inflammatory disorders through prorenin receptors, which activate $\mathrm{p} 38$ MAPK and p42/p44 MAPK. Prorenin activation is completed by both cathepsin B and cathepsin D (Sun et al. 2017). Padmanabhan et al. illustrated that cathepsin B is regarded as a critical cellular protease that activates trimming of SARS-CoV-2 SP and enhances viral entry. Therefore, cathepsin B inhibitors such as hydroxychloroquine may attenuate SARS-CoV-2 infection and RAS activation (Padmanabhan et al. 2020).

Regarding clinical viewpoints, it has been shown that hospitalized Covid-19 patients with HF are at higher risk for development of complications, poor clinical outcomes, and high mortality. Nearly 1 in 4 four hospitalized Covid19 patients with HF died during hospitalization (Bhatt et al. 2021). However, later in pandemic, the risk of mortality for Covid-19 patients with HF are reduced, which could be due to improvement of health system efficiencies and expanded testing capabilities (Horby et al. 2020). Moreover, associated comorbidities such as diabetes mellitus, hypertension, and ischemic heart diseases may augment risk for development of HF during SARS-CoV-2 infection (Phelps et al. 2021). However, a cohort-observational study revealed that hospitalized Covid-19 patients with HF had similar clinical outcomes compared with Covid-19 patients without HF. 
Though, during intensive care unit stay, Covid-19 patients with HF had more in hospital death (Badreldin et al. 2021). The susceptibility of patients with HF for more pronounced complications during development of Covid-19 could be related to injured heart-blood barrier. Leakage of heartblood barrier is induced by matrix metalloproteinase 9 (MMP-9), which provoked by SARS-CoV-2 causing more cardiomyocyte injury (Tyagi and Singh 2021).

Therefore, Covid-19-induced HF is more complex and related to the pro-inflammatory cytokines and neuro-metabolic imbalances that together affect cardiomyocyte functions and development of HF (Fig. 2).

\section{Anti-heart failure drugs and Covid-19}

Digoxin is a common drug used in managing HF and atrial fibrillation; it inhibits $\mathrm{Na} 2+/ \mathrm{K}+$ ATPase in the heart, with subsequent increase cardiomyocyte $\mathrm{Ca} 2+$, which increases cardiac contractility (Lopes et al. 2018). It has been reported that $\mathrm{Na} 2+/ \mathrm{K}+$ ATPase is involved in different RNA and DNA viral infections such as influenza $\mathrm{H} 1 \mathrm{~N} 1$, which reduce expression of $\mathrm{Na} 2+/ \mathrm{K}+$ ATPase in the alveolar epithelial cells (Ulug et al. 1996). However, enterovirus 71 (EV71) increases the expression of $\mathrm{Na} 2+/ \mathrm{K}+$ ATPase through interaction with the $\beta 3$ subunit of this enzyme (Ulug et al. 1996).

The antiviral mechanisms of digoxin and other cardiac glycosides are through inhibiting the synthesis of viral proteins, blocking the viral life cycle, inhibiting pre-mRNA splicing, inhibiting the synthesis of structural proteins, and inhibiting entry of coronaviruses by blocking endocytosis (Lu et al. 2016; Amarelle and Lecuona 2018). In SARSCoV-2 infection, cardiac glycosides including digoxin and ouabain have potent in vitro killing of SARS-CoV-2 more than $99 \%$ compared to negative control and chloroquine (Cho et al. 2020). Digoxin had antiviral and anti-inflammatory effects against SARS-CoV and MERS-CoV through inhibition of mRNA transcription, protein translation, and release of viral particles (Burkard et al. 2015). However, Siniorakis et al. proposed that use of digoxin in Covid-19 may be associated with high mortality due to complicated electrolyte disturbance and acute renal failure (Siniorakis et al. 2021). Therefore, the anti-inflammatory effects of digoxin may inhibit the development of cytokine storms in SARS-CoV-2 infection since Pollard et al. showed that digoxin attenuates cytokine storms in the influenza epidemic (Pollard et al. 2020). Herein, digoxin is recommended to manage atrial fibrillation in patients with severe Covid-19 (Hu et al. 2020). Of note, a combination of digoxin with doxycycline and melatonin might be a promising combination against Covid-19 (Athanasios et al. 2020). The primary mechanism of digoxin anti-inflammatory effect is the suppression of pro-inflammatory cytokines through the NF- $\mathrm{KB}$ inhibition-dependent pathway (Ihenetu et al. 2008). Further, digoxin regulates the unregulated production of immunoglobulins and $\mathrm{T}$ cell activations via induction of regulatory $\mathrm{T}$ cells (Lee et al. 2015). Thus, digoxin may reduce autoimmune myocarditis-induced cardiomyocyte injury and the

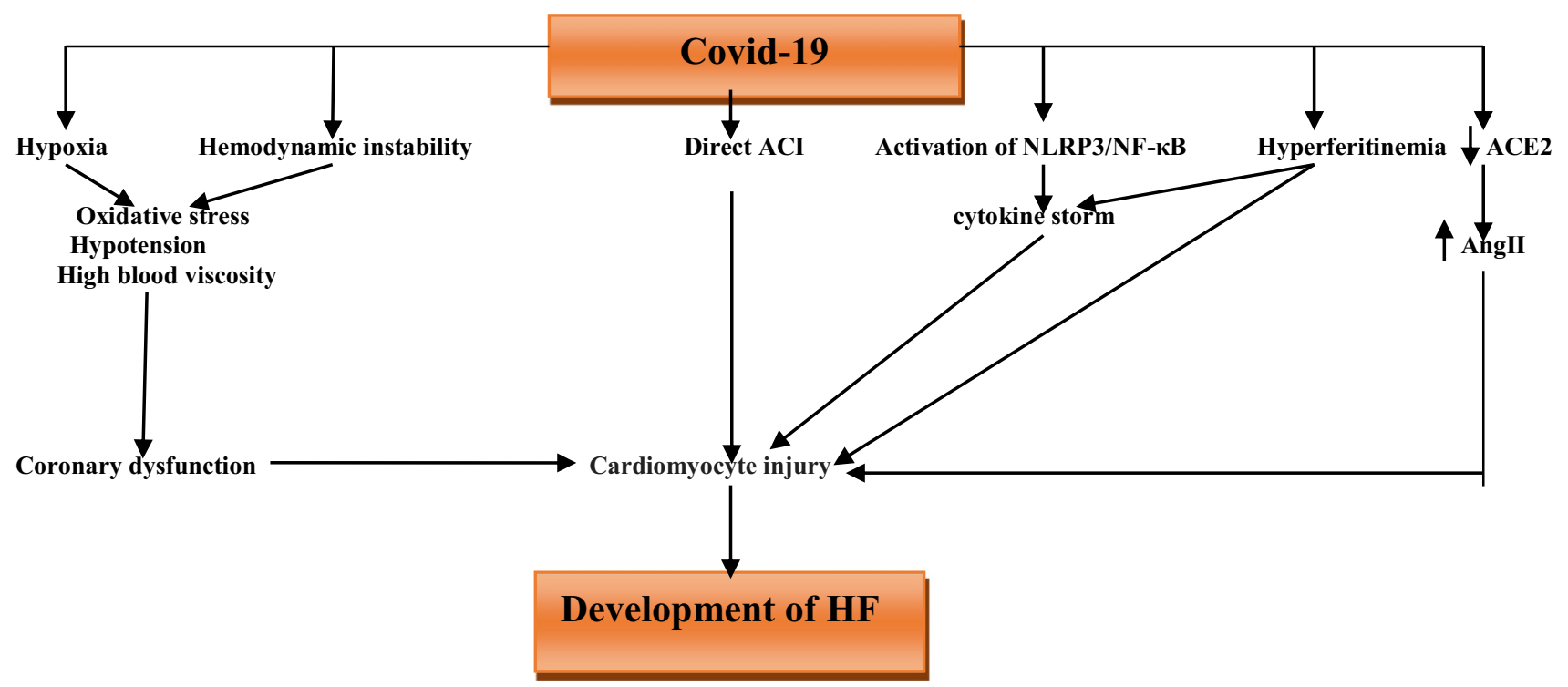

Fig. 2 The association between Covid-19 and development of heart failure (HF): in Covid-19 hypoxia and hemodynamic instability lead to hypotension, oxidative stress and high blood viscosity may cardiomyocyte injury through induction of coronary dysfunction. As well, SARS-CoV-2 may cause direct acute myocardial injury (ACI) and development of HF. In addition, hyperferritinemia and activation of NLRP3/NF- $\kappa$ B signaling pathway contribute into ACI through induction of cytokine storm. Besides, downregulation of ACE2 by SARSCoV-2 upregulates circulating AngII, which involved in cardiomyocyte injury and development of $\mathrm{HF}$ 
development of HF. Nevertheless, a high dose of digoxin may induce local myocardium release of pro-inflammatory cytokine and aggravate myocardial injury in viral myocarditis (Matsumori et al. 1999). The potential role of digoxin in Covid-19 is mainly related to the antiviral and anti-inflammatory effects (Fig. 3).

Indeed, carvedilol, a non-selective $\beta$ adrenoceptor antagonist, and $\alpha-1$ adrenoceptor antagonist, which used to manage congestive HF and left ventricle dysfunction, have a recent activity against SARS-CoV-2 infection (Skayem and Ayoub 2020). Carvedilol downregulates cardiac ACE2 and inhibits SARS-CoV-2-induced acute cardiac injury (Skayem and Ayoub 2020). In addition, carvedilol blocks release and action of IL-6 (Kurum et al. 2007), which involved in tissue injury and the development of cytokine storms. Amirshahrokhi et al. illustrated that carvedilol can attenuate the development of paraquat-induced ALI through suppression of oxidative stress and NF- $\kappa B$ signaling pathway (Amirshahrokhi and Khalili 2016). Also, carvedilol effectively manages Covid-19 complications such as esophageal varices (Congly et al. 2020) and postCovid-19 sinus tachycardia (Kartik Pandurang and Pankaj 2020). Therefore, through its antiviral and anti-inflammatory properties, carvedilol may have dual protective effects in Covid-19 by mitigation development of HF and ALI (Servato et al. 2021).

Moreover, mineralocorticoid receptor antagonists, like eplerenone and spironolactone that are used in the management of HF, have anti-SARS-CoV-2 effects and prevent acute complications of Covid-19, including HF and ALI (Kotfis et al. 2021). Likewise, mineralocorticoid antagonists attenuate the development of ALI, ARDS, and pulmonary hypertension in severe Covid-19 through regulation of RAS (Dumanll et al. 2020).

Furthermore, vasopressin receptor antagonists such as conivaptan have in silico anti-SARS-CoV-2 to inhibit RNA-dependent polymerase and non-structural protein 9 (Gul et al. 2020). Besides, conivaptan inhibits release of pro-inflammatory cytokines (Can et al. 2018); thus, it can be a potential candidate against SARS-CoV-2 infection and linked inflammatory disorders.

Taken together, medications that are used in the management of HF like digoxin and adrenoceptor antagonists during the development of Covid-19 should be revised as some drugs could have dual properties against HF and SARS-CoV-2 infection in patients with severe Covid-19.
Fig. 3 Role of digoxin in the attenuation Covid-19 inducedheart failure. Digoxin inhibits replication of SARS-CoV-2 and NF- $\kappa B$, thereby inhibiting cytokine storm-induced heart failure

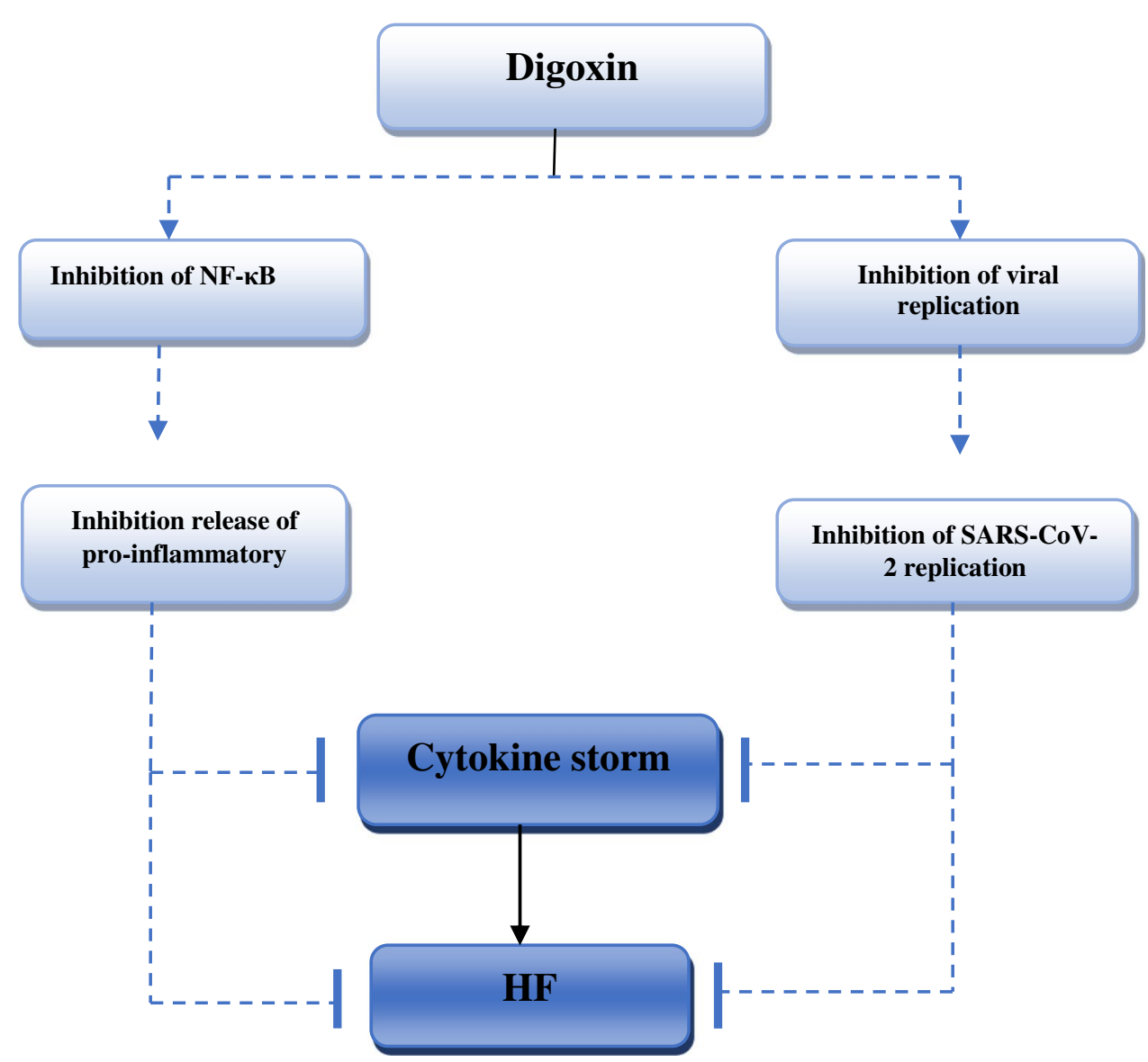




\section{Conclusion}

SARS-CoV-2 infection may lead to the development of HF due to direct acute cardiac injury or through progression of cytokine storms, which depress cardiomyocyte function and cardiac contractility. Anti-heart failure drugs, mainly digoxin and carvedilol, may attenuate development of HF by reducing the infectivity of SARS-CoV-2 and prevent the development of cytokine storms in severely affected Covid-19 patients.

Author contribution HMA, HO, AIA, SQ, EMA, and GEB conceived and designed research. HMA, HO, and AIA conducted experiments. HMA, HO, AIA, SQ, EMA, and GEB AM and GR analyzed the graphical illustrations data. HMA, HO, AIA, and GEB wrote the manuscript. All authors read and approved the manuscript and all data were generated in-house and that no paper mill was used.

Data availability The datasets/information used for this study are available from the corresponding author on reasonable request.

\section{Declarations}

\section{Ethics approval and consent to participate Not applicable}

Consent for publication All the authors have read and agreed to the final copy of the finding as contained in the manuscript.

Competing interests The authors declare no competing interests.

\section{References}

Adinolfi LE, Zampino R, Restivo L, et al. (2014) Chronic hepatitis $\mathrm{C}$ virus infection and atherosclerosis: clinical impact and mechanisms. World J Gastroenterolhttps://doi.org/10.3748/wjg. v20.i13.3410

Al-kuraishy H, Al-Gareeb A, Al-Buhadilly A (2018) Rosuvastatin improves vaspin serum levels in obese patients with acute coronary syndrome. Diseaseshttps://doi.org/10.3390/diseases60 10009

Al-Kuraishy H, Hussien N, Al-Naimi M, et al. (2020) Is ivermectinazithromycin combination the next step for COVID-19? Biomed Biotechnol Res Jhttps://doi.org/10.4103/bbrj.bbrj_109_20

Al-Kuraishy HM, Al-Gareeb AI (2017) Acylation-stimulating protein is a surrogate biomarker for acute myocardial infarction: role of statins. J Lab Physicianshttps://doi.org/10.4103/0974-2727. 208263

Al-Kuraishy HM, Al-Gareeb AI (2016) Potential effects of pomegranate on lipid peroxidation and pro-inflammatory changes in daunorubicin-induced cardiotoxicity in rats. Int J Prev Medhttps:// doi.org/10.4103/2008-7802.184314

Al-kuraishy HM, Al-Gareeb AI, Abdullah SM et al (2021) Case report: Hyperbilirubinemia in Gilbert syndrome attenuates Covid-19-induced metabolic disturbances. Front Cardiovasc Med. https://doi. org/10.3389/fcvm.2021.642181

Al-kuraishy HM, Al-Gareeb AI, Alblihed M et al (2021) COVID-19 and risk of acute ischemic stroke and acute lung injury in patients with type II diabetes mellitus: the anti-inflammatory role of metformin. Front Med. https://doi.org/10.3389/fmed.2021.644295

Al-Kuraishy HM, Al-Gareeb AI, Almulaiky YQ, Cruz-Martins N, \& Batiha GES (2021) Role of leukotriene pathway and montelukast in pulmonary and extrapulmonary manifestations of Covid-19: The enigmatic entity. European journal of pharmacology, 174196

Al-kuraishy HM, Al-Gareeb AI, Alzahrani KJ et al (2021) The potential role of neopterin in Covid-19: a new perspective. Mol Cell Biochem. https://doi.org/10.1007/s11010-021-04232-z

Al-kuraishy HM, Al-Gareeb AI, Faidah H, et al. (2021e) The looming effects of estrogen in Covid-19: a rocky rollout. Front Nutr

Amarelle L, Lecuona E (2018) The antiviral effects of Na,K-ATPase inhibition: a minireview. Int J Mol Sci

Amirshahrokhi K, Khalili AR (2016) Carvedilol attenuates paraquatinduced lung injury by inhibition of proinflammatory cytokines, chemokine MCP-1, NF- $\mathrm{\kappa B}$ activation and oxidative stress mediators. Cytokine https://doi.org/10.1016/j.cyto.2016.09.004

Ashraf H, Norouzi P, Jafari S (2011) Hodgkin's disease with hyperferritinemia, hepatic and heart failure. Govaresh

Asiedu SO, Kwofie SK, Broni E, Wilson MD (2021) Computational identification of potential anti-inflammatory natural compounds targeting the p38 mitogen-activated protein kinase (Mapk): implications for covid-19-induced cytokine storm. Biomolecules. https://doi.org/10.3390/biom11050653

Athanasios T, Psychos C, Domenikos S (2020) Communication: Novel drug combination doxycycline-melatonin-digoxin(D-M-D) for possible Covid-19 treatment. J Mod Med Chem https://doi.org/ 10.12970/2308-8044.2020.08.01

Bader F, Manla Y, Atallah B, Starling RC (2021) Heart failure and COVID-19. Heart Fail. Rev

Badreldin H, Hafidh D, Bin Saleh D et al (2021) Clinical characteristics and outcomes of patients with heart failure admitted to the intensive care unit with coronavirus disease 2019 (COVID19): a multicenter cohort study. Eur J Cardiovasc Nurs. https://doi.org/ 10.1093/eurjen/zvab060.041

Belhadjer Z, Méot M, Bajolle F et al (2020) Acute heart failure in multisystem inflammatory syndrome in children in the context of global SARS-CoV-2 pandemic. Circulation. https://doi.org/10. 1161/CIRCULATIONAHA. 120.048360

Bhatt AS, Jering KS, Vaduganathan $M$ et al (2021) Clinical outcomes in patients with heart failure hospitalized with COVID-19. JACC Hear Fail. https://doi.org/10.1016/j.jchf.2020.11.003

Bozkurt B, Kovacs R, Harrington B (2020) Joint HFSA/ACC/AHA statement addresses concerns re: using RAAS antagonists in COVID-19. J Card Fail

Burkard C, Verheije MH, Haagmans BL et al (2015) ATP1A1-mediated Src signaling inhibits coronavirus entry into host cells. J Virol. https://doi.org/10.1128/jvi.03274-14

Butt AA, Chang CC, Kuller L et al (2011) Risk of heart failure with human immunodeficiency virus in the absence of prior diagnosis of coronary heart disease. Arch Intern Med. https://doi.org/10. 1001/archinternmed.2011.151

Butts B, Gary RA, Dunbar SB, Butler J (2015) The importance of NLRP3 inflammasome in heart failure. J Card Fail

Can B, Öz S, Muşmul A, et al (2018) Effects of conivaptan and mannitol on serum cytokine levels (TNF- $\alpha$, IL-15 and IL-35) following bilateral carotid artery occlusion. undefined

Chen C, Zhou Y, Wang DW (2020a) SARS-CoV-2: a potential novel etiology of fulminant myocarditis. Herz

Chen L, Hao G (2020) The role of angiotensin-converting enzyme 2 in coronaviruses/influenza viruses and cardiovascular disease. Cardiovasc Res

Chen L, Li X, Chen M et al (2020) The ACE2 expression in human heart indicates new potential mechanism of heart injury among patients infected with SARS-CoV-2. Cardiovasc Res. https://doi. org/10.1093/CVR/CVAA078 
Cho J, Lee YJ, Kim JH et al (2020) Antiviral activity of digoxin and ouabain against SARS-CoV-2 infection and its implication for COVID-19. Sci Rep. https://doi.org/10.1038/s41598-020-72879-7

Congly SE, Sadler MD, Abraldes JG et al (2020) Practical management of esophageal varices in the context of SARS-CoV-2 (COVID19): the Alberta protocol. Can Liver J. https://doi.org/10.3138/ canlivj-2020-0007

Dumanlı GY, Dilken O, Ürkmez S (2020) Use of spironolactone in SARS-CoV-2 ARDS patients. Turk J Anaesthesiol Reanim

Falsey AR, Walsh EE, Esser MT et al (2019) Respiratory syncytial virus-associated illness in adults with advanced chronic obstructive pulmonary disease and/or congestive heart failure. J Med Virol. https://doi.org/10.1002/jmv.25285

Farré N, Vela E, Clèries M et al (2017) Real world heart failure epidemiology and outcome: a population-based analysis of 88,195 patients. PLoS One. https://doi.org/10.1371/journal.pone.0172745

Gul S, Ozcan O, Asar S et al (2020) In silico identification of widely used and well-tolerated drugs as potential SARS-CoV-2 3C-like protease and viral RNA-dependent RNA polymerase inhibitors for direct use in clinical trials. J Biomol Struct Dyn. https://doi.org/ 10.1080/07391102.2020.1802346

Han H, Ma Q, Li C et al (2020) Profiling serum cytokines in COVID19 patients reveals IL- 6 and IL- 10 are disease severity predictors. Emerg Microbes Infect. https://doi.org/10.1080/22221751.2020. 1770129

Hemmat N, Asadzadeh Z, Ahangar NK, et al (2021) The roles of signaling pathways in SARS-CoV-2 infection; lessons learned from SARS-CoV and MERS-CoV. Arch Virol

Horby P, Lim WS, Emberson J et al (2020) Effect of dexamethasone in hospitalized patients with COVID-19-preliminary report. N Engl J Med. https://doi.org/10.1101/2020.06.22.20137273

Hu YF, Cheng WH, Hung Y, et al. (2020) Management of atrial fibrillation in COVID-19 pandemic. Circ J

Ihenetu K, Espinosa R, De Leon R et al (2008) Digoxin and digoxinlike immunoreactive factors (DLIF) modulate the release of proinflammatory cytokines. Inflamm Res. https://doi.org/10.1007/ s00011-008-7249-9

Imazio M, Klingel K, Kindermann I, et al. (2020) COVID-19 pandemic and troponin: indirect myocardial injury, myocardial inflammation or myocarditis? Heart

Kartik Pandurang J, Pankaj V J (2020) Ivabradine versus carvedilol in the management of palpitation with sinus tachycardia among recovered COVID-19 patients. J Cardiol Cardiovasc Med https:// doi.org/10.29328/journal.jccm.1001107

Keidar S, Gamliel-Lazarovich A, Kaplan M et al (2005) Mineralocorticoid receptor blocker increases angiotensin-converting enzyme 2 activity in congestive heart failure patients. Circ Res. https://doi. org/10.1161/01.RES.0000187500.24964.7A

Kotfis K, Lechowicz K, Drożdżal S, et al (2021) COVID-19-the potential beneficial therapeutic effects of spironolactone during SARS-CoV-2 infection. Pharmaceuticals

Kristoffersen US, Lebech AM, Gerstoft J et al (2008) Right and left cardiac function in HIV-infected patients investigated using radionuclide ventriculography and brain natriuretic peptide: a 5-year follow-up study. HIV Med. https://doi.org/10.1111/j.1468-1293. 2007.00531.x

Kurum T, Tatli E, Yuksel M (2007) Effects of carvedilol on plasma levels of pro-inflammatory cytokines in patients with ischemic and nonischemic dilated cardiomyopathy. Texas Hear Inst J

Lee J, Baek S, Lee J et al (2015) Digoxin ameliorates autoimmune arthritis via suppression of Th17 differentiation. Int Immunopharmacol. https://doi.org/10.1016/j.intimp.2015.03.017

Liang J, Huang B, Yuan G, et al (2017) Stretch-activated channel Piezo1 is up-regulated in failure heart and cardiomyocyte stimulated by Angii. Am J Transl Res
Lin MS, Chung CM, Chang ML et al (2018) The unraveled link between antiviral therapy and heart failure hospitalization in chronic hepatitis c virus infection-a nationwide cohort study. Circ J. https://doi.org/10.1253/circj.CJ-17-1118

Lopes RD, Rordorf R, De Ferrari GM et al (2018) Digoxin and mortality in patients with atrial fibrillation. J Am Coll Cardiol. https:// doi.org/10.1016/j.jacc.2017.12.060

Lovren F, Pan Y, Quan A et al (2008) Angiotensin converting enzyme-2 confers endothelial protection and attenuates atherosclerosis. Am J Physiol - Heart Circ Physiol. https://doi.org/10.1152/ajpheart. 00331.2008

Lu Y, Hou H, Wang F et al (2016) ATP1B3: a virus-induced host factor against EV71 replication by up-regulating the production of type-I interferons. Virology. https://doi.org/10.1016/j.virol.2016.05.013

Lugnier C, Al-Kuraishy HM, Rousseau E (2021) PDE4 inhibition as a therapeutic strategy for improvement of pulmonary dysfunctions in Covid-19 and cigarette smoking. Biochem Pharmacol

M. Al-Kuraishy H, S. Al-Niemi M, R. Hussain N, et al (2020) The potential role of renin angiotensin system (RAS) and dipeptidyl peptidase-4 (DPP-4) in COVID-19: navigating the uncharted. In: Selected chapters from the renin-angiotensin system

Markousis-Mavrogenis G, Tromp J, Ouwerkerk W et al (2019) The clinical significance of interleukin-6 in heart failure: results from the BIOSTAT-CHF study. Eur J Heart Fail. https://doi.org/10. 1002/ejhf.1482

Matsoukas J, Apostolopoulos V, Zulli A, et al (2021) From angiotensin II to cyclic peptides and angiotensin receptor blockers (Arbs): perspectives of arbs in covid-19 therapy. Molecules

Matsumori A, Igata H, Ono K et al (1999) High doses of digitalis increase the myocardial production of proinflammatory cytokines and worsen myocardial injury in viral myocarditis: a possible mechanism of digitalis toxicity. Jpn Circ J. https://doi.org/10. 1253/jcj.63.934

Matsumori A, Shimada T, Chapman NM et al (2006) Myocarditis and heart failure associated with hepatitis $\mathrm{C}$ virus infection. J Card Fail. https://doi.org/10.1016/j.cardfail.2005.11.004

Meijles DN, Cull JJ, Markou T et al (2020) Redox regulation of cardiac ASK1 (apoptosis signal-regulating kinase 1) controls p38MAPK (mitogen-activated protein kinase) and orchestrates cardiac remodeling to hypertension. Hypertension. https://doi.org/ 10.1161/HYPERTENSIONAHA. 119.14556

Moubarak M, Kasozi KI, Hetta HF et al (2021) The rise of SARS$\mathrm{CoV}-2$ variants and the role of convalescent plasma therapy for management of infections. Life. https://doi.org/10.3390/life1 1080734

Nico Reinsch, Neuhaus Kathrin, Esser Stefan, Potthoff Anja, Hower Martin, Brockmeyer Norbert H, Raimund Erbel TN (2010) Prevalence of cardiac diastolic dysfunction in HIV-infected patients: results of the HIV-HEART study. HIV Clin Trials 11:156-162. https://doi.org/10.1310/HCT1103-156

Noroozi R, Branicki W, Pyrc K, et al (2020) Altered cytokine levels and immune responses in patients with SARS-CoV-2 infection and related conditions. Cytokine

Ohtsuki M, Morimoto SI, Izawa H et al (2010) Angiotensin converting enzyme 2 gene expression increased compensatory for left ventricular remodeling in patients with end-stage heart failure. Int $\mathrm{J}$ Cardiol. https://doi.org/10.1016/j.ijcard.2009.11.057

Oudit GY, Kassiri Z, Jiang C et al (2009) SARS-coronavirus modulation of myocardial ACE2 expression and inflammation in patients with SARS. Eur J Clin Invest. https://doi.org/10.1111/j.13652362.2009.02153.x

Padmanabhan P, Desikan R, Dixit NM (2020) Targeting TMPRSS2 and cathepsin $\mathrm{B} / \mathrm{L}$ together may be synergistic against SARSCoV-2 infection. ChemRxiv. https://doi.org/10.26434/chemrxiv. 12213125.v2 
Panhwar MS, Kalra A, Gupta T et al (2019) Effect of influenza on outcomes in patients with heart failure. JACC Hear Fail. https:// doi.org/10.1016/j.jchf.2018.10.011

Patel AB, Verma A (2020) COVID-19 and angiotensin-converting enzyme inhibitors and angiotensin receptor blockers: what is the evidence? JAMA - J Am Med Assoc

Peng X, Wang Y, Xi X, et al. (2021) Promising therapy for heart failure in patients with severe COVID-19: calming the cytokine storm. Cardiovasc Drugs Ther

Phelps M, Christensen DM, Gerds T et al (2021) Cardiovascular comorbidities as predictors for severe COVID-19 infection or death. Eur Hear J - Qual Care Clin Outcomes. https://doi.org/10. 1093/ehjqcco/qcaa081

Pollard BS, Blancol JC, Pollard JR (2020) Classical drug digitoxin inhibits influenza cytokine storm, with implications for Covid19 therapy. In Vivo (Brooklyn). https://doi.org/10.21873/invivo. 12221

Reza N, DeFilippis EM, Jessup M (2020) Secondary impact of the COVID-19 pandemic on patients with heart failure. Circ Hear Fail

Rossignol P, Hernandez AF, Solomon SD, Zannad F (2019) Heart failure drug treatment. Lancet

Saed Aldien A, Ganesan GS, Wahbeh F, et al (2021) Systemic inflammation may induce cardiac injury in COVID-19 patients including children and adolescents without underlying cardiovascular diseases: a systematic review. Cardiovasc. Revascularization Med.

Sanchis-Gomar F, Lavie CJ, Perez-Quilis C, et al (2020) Angiotensinconverting enzyme 2 and antihypertensives (angiotensin receptor blockers and angiotensin-converting enzyme inhibitors) in coronavirus disease 2019. Mayo Clin Proc

Servato ML, Valente FX, García-Moreno LG et al (2021) Intraventricular conundrum in a SARS-CoV-2-positive patient with elevated biomarkers of myocardial injury. JACC Case Rep. https://doi.org/ 10.1016/j.jaccas.2021.01.030

Siniorakis E, Arvanitakis S, Katsianis A, Elkouris M (2021) Atrial fibrillation and flutter in patients hospitalized for COVID-19: the challenging role of digoxin. J Cardiovasc Electrophysiol

Skayem C, Ayoub N (2020) Carvedilol and COVID-19: a potential role in reducing infectivity and infection severity of SARS-CoV-2. Am J Med Sci
Soto M, Bang SI, McCombs J, Rodgers KE (2017) Renin Angiotensin system-modifying therapies are associated with improved pulmonary health. Clin Diabetes Endocrinol. https://doi.org/10.1186/ s40842-017-0044-1

Sun Y, Danser AHJ, Lu X (2017) (Pro)renin receptor as a therapeutic target for the treatment of cardiovascular diseases? Pharmacol Res

Taz TA, Ahmed K, Paul BK et al (2021) Identification of biomarkers and pathways for the SARS-CoV-2 infections that make complexities in pulmonary arterial hypertension patients. Brief Bioinform. https://doi.org/10.1093/bib/bbab026

Tsui JI, Whooley MA, Monto A et al (2009) Association of hepatitis C virus seropositivity with inflammatory markers and heart failure in persons with coronary heart disease: data from the Heart and Soul Study. J Card Fail. https://doi.org/10.1016/j.cardfail.2008. 12.003

Tyagi SC, Singh M (2021) Multi-organ damage by covid-19: congestive (cardio-pulmonary) heart failure, and blood-heart barrier leakage. Mol Cell Biochem

Ulug ET, Garry RF, Bose HR (1996) Inhibition of Na+K+ATPase activity in membranes of Sindbis virus-infected chick cells. Virology. https://doi.org/10.1006/viro.1996.0065

Vardeny O, Solomon SD (2019) Influenza and heart failure: a catchy comorbid combination. JACC Hear Fail

VasanthiDharmalingam P, Karuppagounder V, Watanabe K, et al (2021) SARS-CoV-2 mediated hyperferritinemia and cardiac arrest: preliminary insights. Drug Discov Today

Wang Y, Tikellis C, Thomas MC, Golledge J (2013) Angiotensin converting enzyme 2 and atherosclerosis. Atherosclerosis

Zhou F, Yu T, Du R et al (2020) Clinical course and risk factors for mortality of adult inpatients with COVID-19 in Wuhan, China: a retrospective cohort study. Lancet. https://doi.org/10.1016/S01406736(20)30566-3

Publisher's note Springer Nature remains neutral with regard to jurisdictional claims in published maps and institutional affiliations. 